\title{
Designing a Low- Pass FIR Digital Filter by using Rectangular Window Hanning Window and Bartlett Method
}

\author{
Vijendra Kumar Prajapati ${ }^{1}$, Dhanmohan Ratre ${ }^{2}$, Pranay Kumar Rahi ${ }^{3}$ \\ B.E. Scholar, Dept of Electrical and Electronics Engg, Institute of Technology, Korba, Chhattisgarh, India ${ }^{1,2}$ \\ Assistant Professor, Dept of Electrical and Electronics Engg, Institute of Technology, Korba, Chhattisgarh, India ${ }^{3}$
}

\begin{abstract}
Digital filtering plays a significant role in the world of technology. Therefore, processing of input signal has to be done to get useful signal. This paper deals with the design of finite impulse response digital filter using window techniques Rectangular, Hanning and Bartlett of order (15) are presented. It shown that filterdesign by using Rectangular Window Technique is better as it provides better result in terms of magnitude, phase, impulse, step responses and pole-zero plot.[1]
\end{abstract}

Keywords: FIR Filter, DSP, FFT, Hanning Window, MATLAB, Rectangular Window, Bartlett Window, frequency sampling, amplitude-frequency.

\section{INTRODUCTION}

Digital signal processing is the technique employed for the mathematical manipulation of an information signal so as to modify or improve it. For this purpose filters are mainly used[2]. The filter is used to describe a linear time-invarient system used to perform spectral shaping or frequency selective filtering. Filter is used in digital signal processing is widely used a number of ways, such as equalization of communication channels, signal detection in radar, sonar and communications, and for performing analysis of the spectra of a variety of signal [3]. A digital filter is a system which passes some desired signals more than others to reduce or enhance certain aspects of that signal. It can be used to pass the signals according to the specified frequency pass-band and reject the other frequency than the pass-band specification [4]

Application of digital signal processing

Military - radar signal processing, sonar signal processing, navigation, secure missile guidance.

Image processing - image representation, image compression, image enhancement, satellite weather map and animation, robotic vision, image analysis recognition

Medicine- Medical diagnostic instrumentation such as computerized tomography (CT), X-ray scanning, Patient monitoring and Xray

Signal filtering- Removing of unwanted background noise, removal of interference, separation of frequency bands and shaping of the signal spectrum.

Telecommunication - Echo cancellation in telephone networks, equalization, telephone dialing application, modems, line repeaters, channel multiplexing, data encryption, video conferencing, cellular phone and FAX [5]

\section{WINDOW TEECHNIQUE}

The FIR filter design process using window function can be enumerated as:

i. Define filter specification

ii. Specify a window function according to the filterspecification.

iii. Compute the filter order required for a given setof specification.

iv. Compute the coefficients of the window functionto be used.

v. Compute the coefficients the ideal filteraccording to the filter order.

vi. Compute FIR filter coefficients in accordance the obtained window function and the coefficients ofthe ideal filter.

vii. If the resulting filter has a very wide or a verynarrow transition region, it is mandatory tochange the filter order by decreasing it accordingto needs, and after this process the steps 4, 5 and6 are interated as many times as needs. 
The window used in this paper to design the FIR filter are:

1.Rectangular window.

2.Hanning

3.Barclett [6]

\subsection{RECTANGULAR WINDOW}

The rectangular window is what you would obtain if you were to simply segment a finite portion of the impulse response without any shaping in the time domain:

$\omega[n]=\{1,0 \leq n \leq M$, otherwise

We have studied this function extensively in class, and know it's DTFT to be $\omega(e j \omega)=\sin (M \omega 2) \sin (\omega 2) e-j \omega M 2 /$

Compare the plots of the original sinc function above (without the phase term) and its magnitude plotted in $\mathrm{dB}$, $20 \log 10(|\omega(e j \omega)|) \cdot[7]$

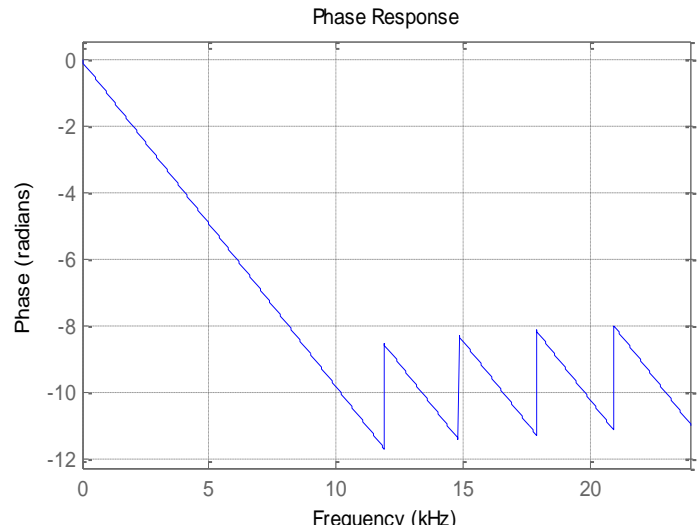

Fig.(1) Phase Respoce of Recantugar window

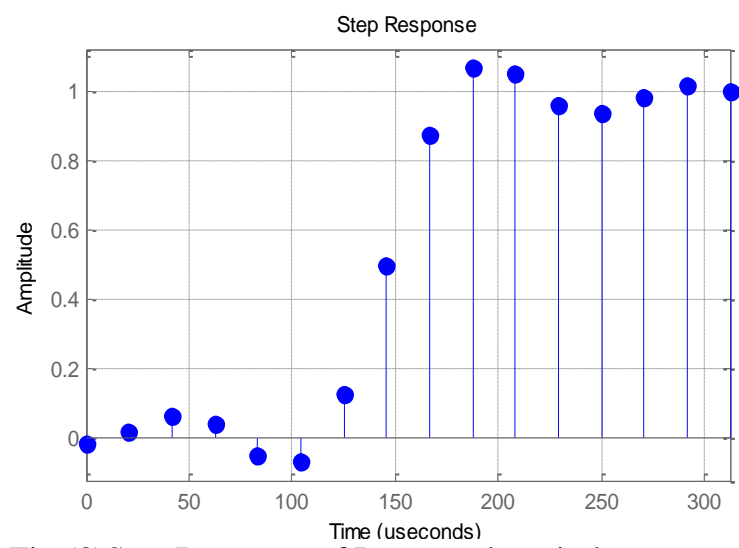

Fig.(3)Step Response of Rectangular window

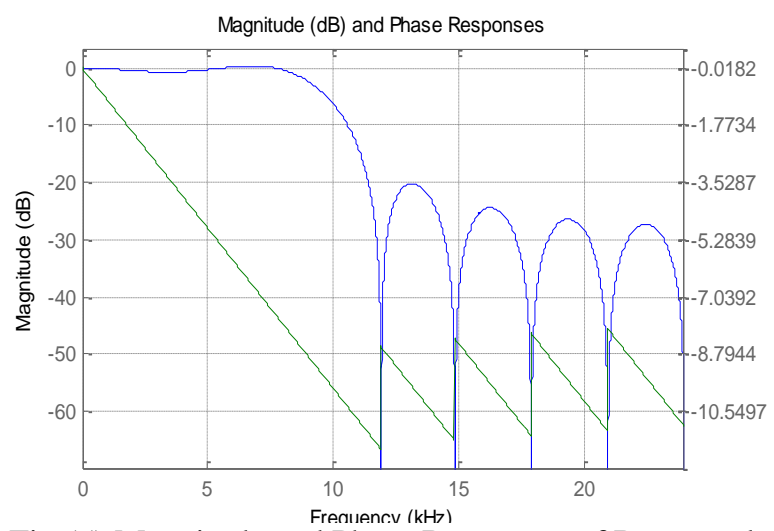

Fig.(5) Magnitude and Phase Responses of Rectangular

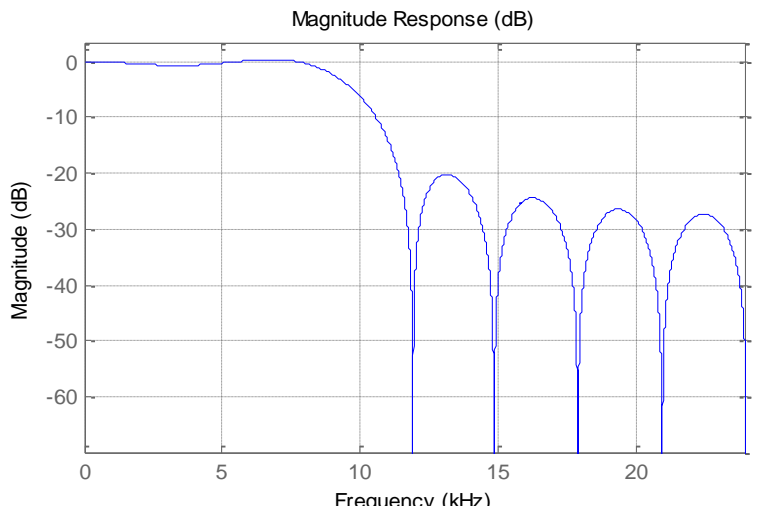

Fig.(2) Magnitude Response of Recantular window

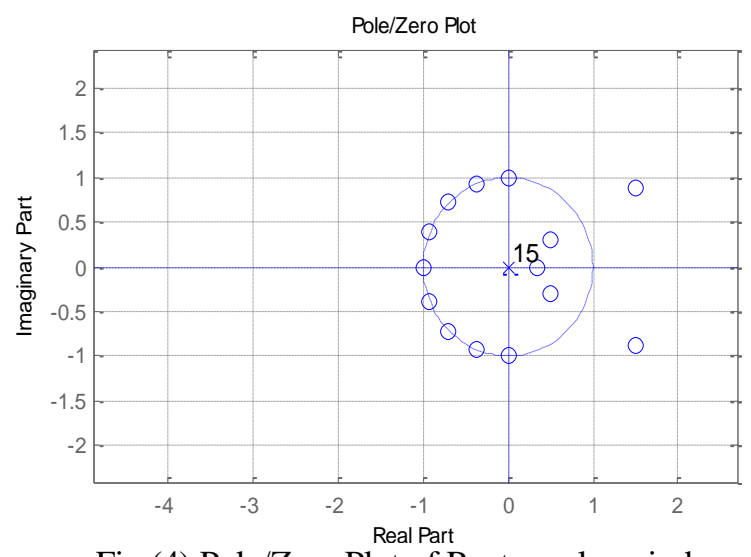

Fig.(4) Pole/Zero Plot of Rectangular window

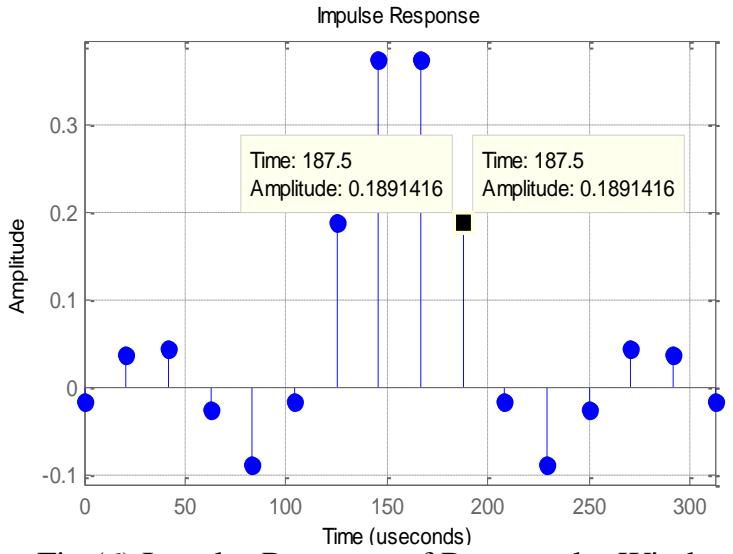

Fig.(6) Impulse Response of Rectangular Window 


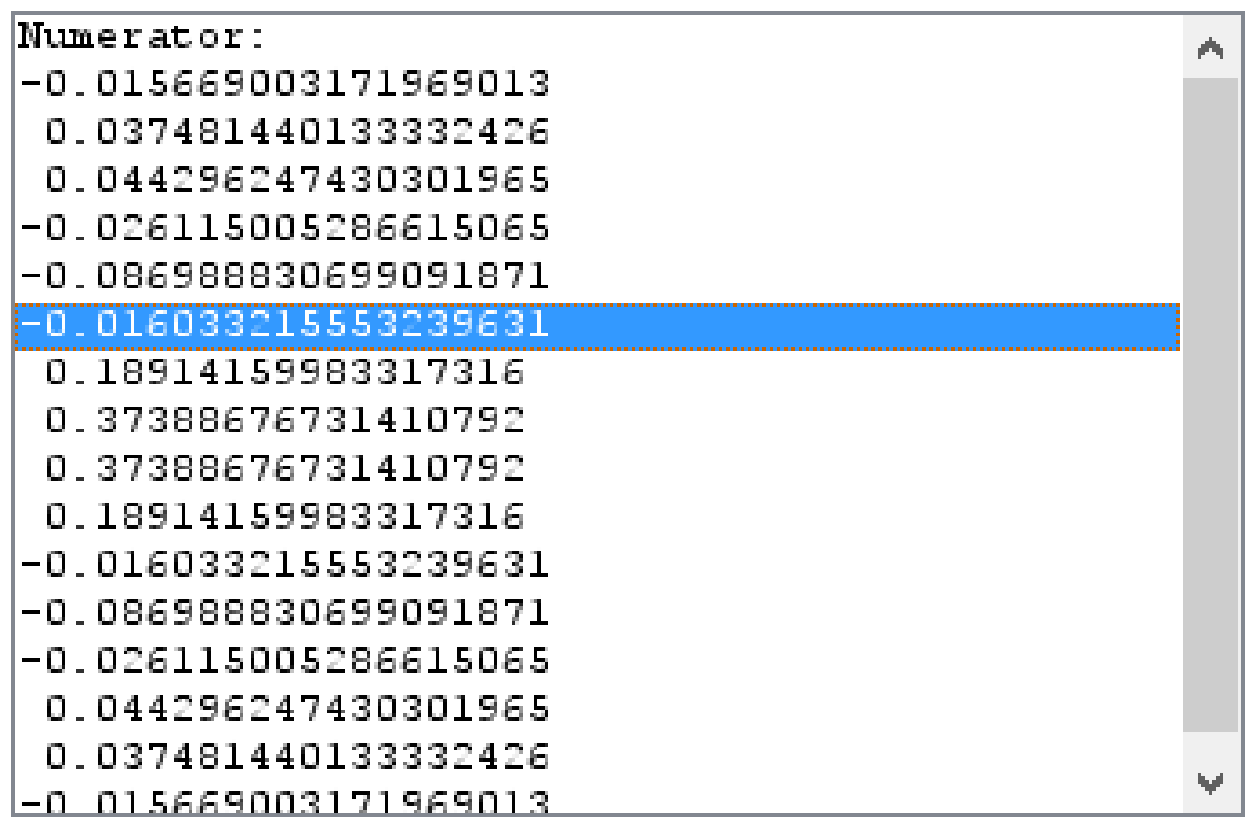

Fig.(7) Coficcient of Rectangular window Tecnique

2.2Hanning window

Syntax: $\mathrm{w}=$ hann $(\mathrm{L})$

Description: It returns an L-point symmetric Hann window in the column vector w. L must be a positive integer. The coefficients of a Hann window are computed from the following equation.
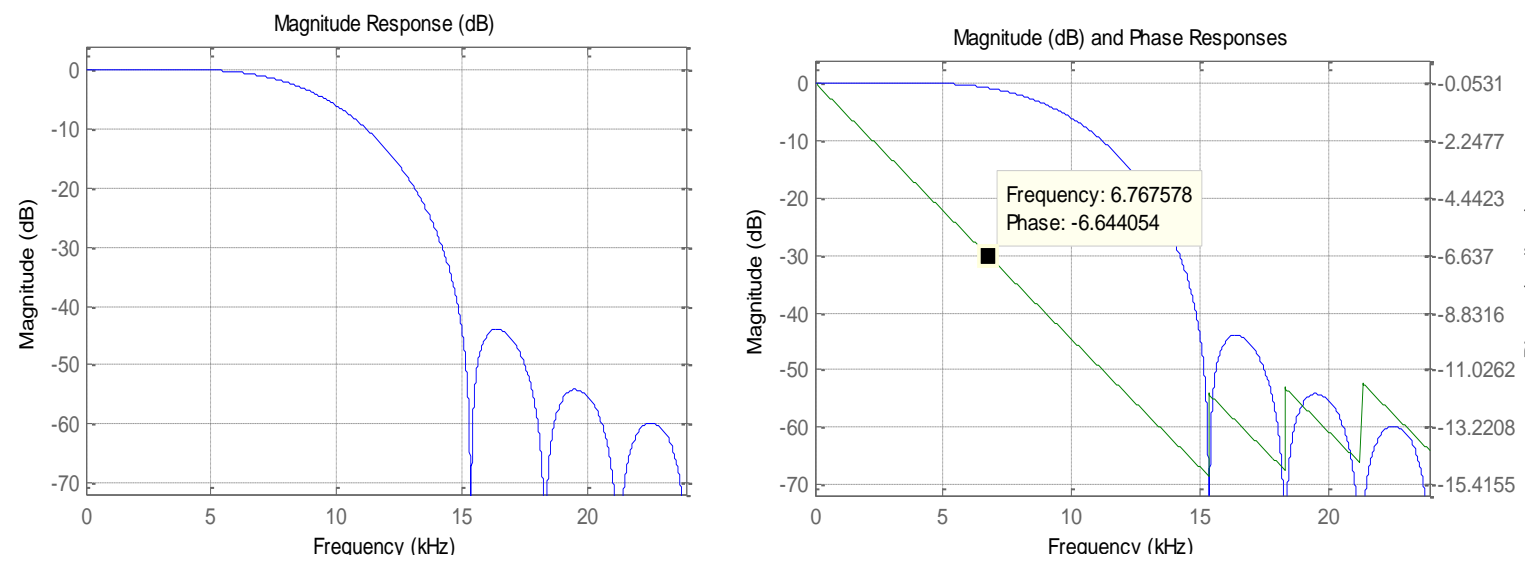

Fig.(8) Magnitude Response of Hanning window

Fig.(9) Mangnitude and Phase Response of Hanning window

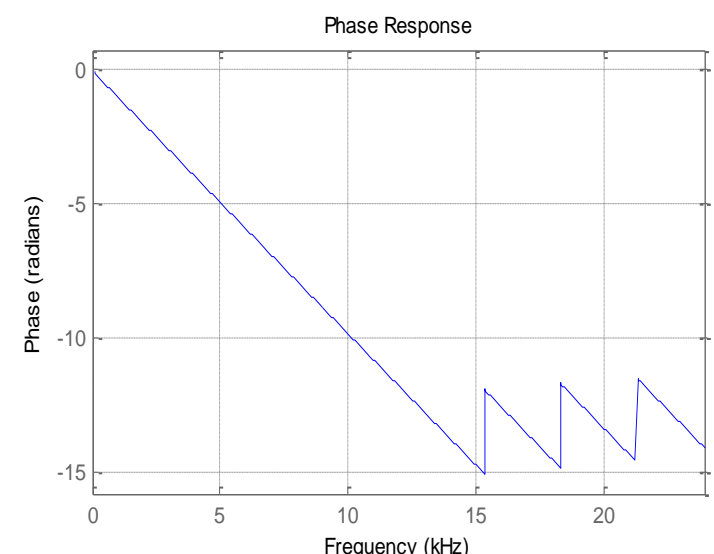

Fig.(10) Phase Response of Hanning window

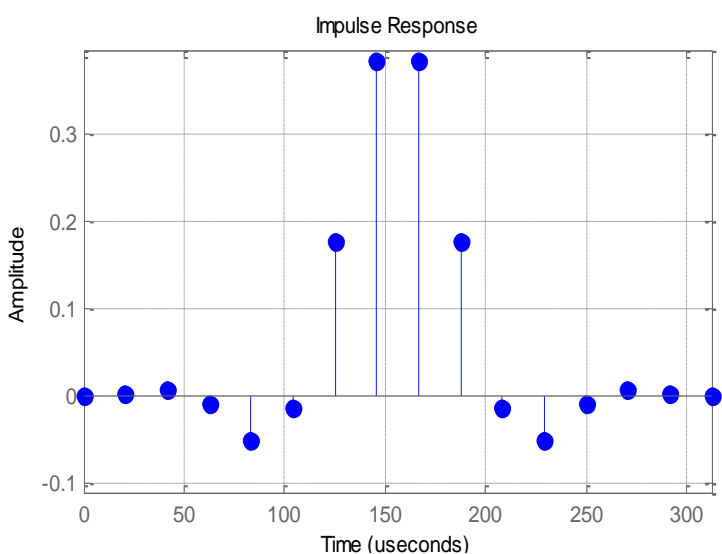

Fig.(11) Impulse Response of Hanning window 


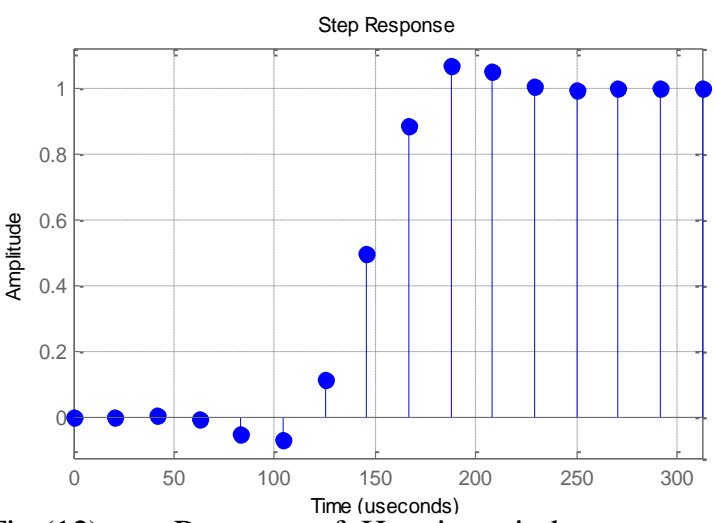

Fig.(12) step Response of Hanning window

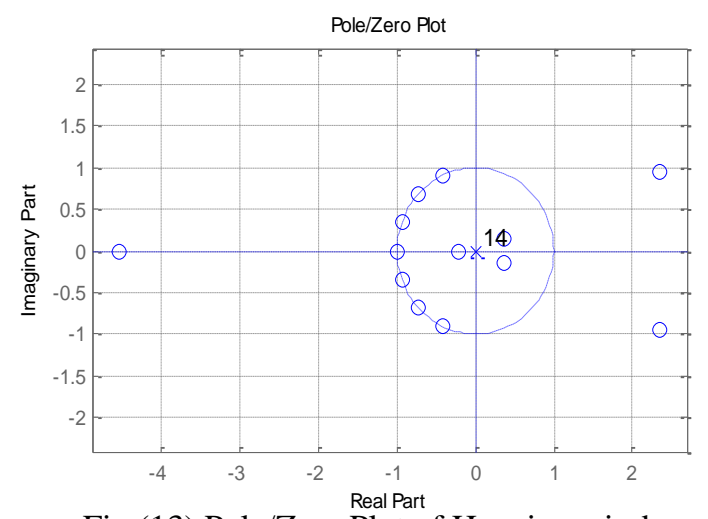

Fig.(13) Pole/Zero Plot of Hanning window

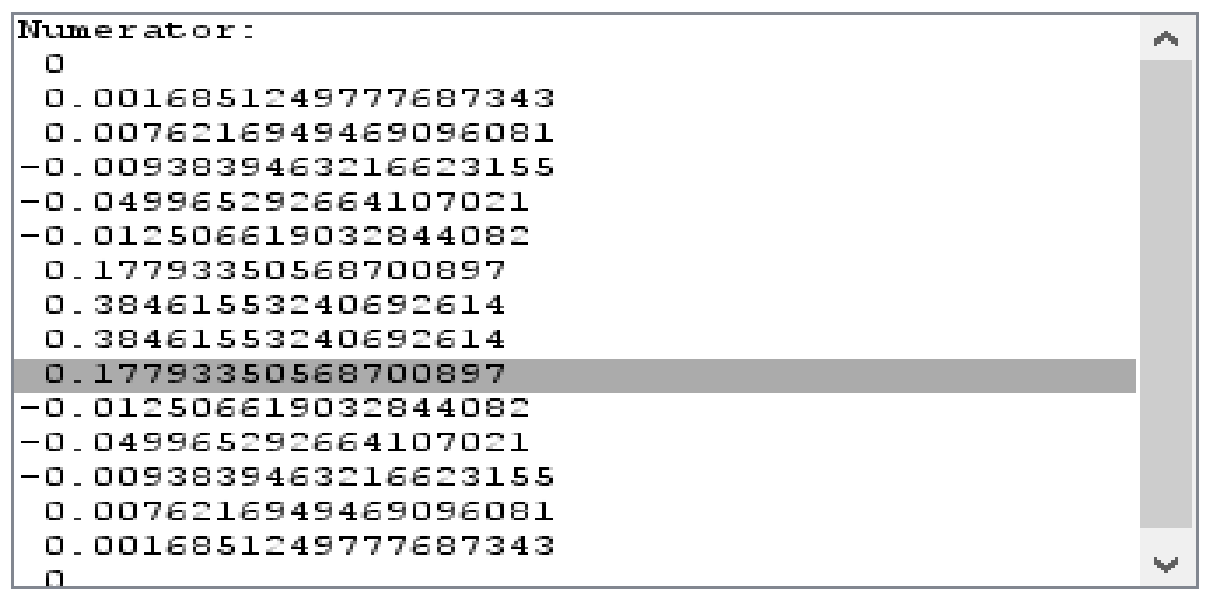

FIG.(14) Filter Coefficant of Hanning window

\subsection{The Bartlett window}

The Bartlett window is very similar to a triangular window as returned by the triang function. The Bartlett window always has zeros at the first and last samples, however, while the triangular window is nonzero at those points. For $\mathrm{L}$ odd, the center $\mathrm{L}-2$ points of bartlett(L) are equivalent to triang(L-2).

$\mathrm{w}=$ bartlett(L) returns an L-point Bartlett window in the column vector $\mathrm{w}$, where L must be a positive integer. The coefficients of a Bartlett window are computed as follows:

( )

$\mathrm{w} \mathrm{n}=_{2 \mathrm{nN}}, 2-{ }_{2 \mathrm{nN}}, 0 \leq \mathrm{n} \leq{ }_{\mathrm{N} 2 \mathrm{~N} 2} \leq \mathrm{n} \leq \mathrm{N}$

$8>><>$ :

The window length $\mathrm{L}=\mathrm{N}+1$.

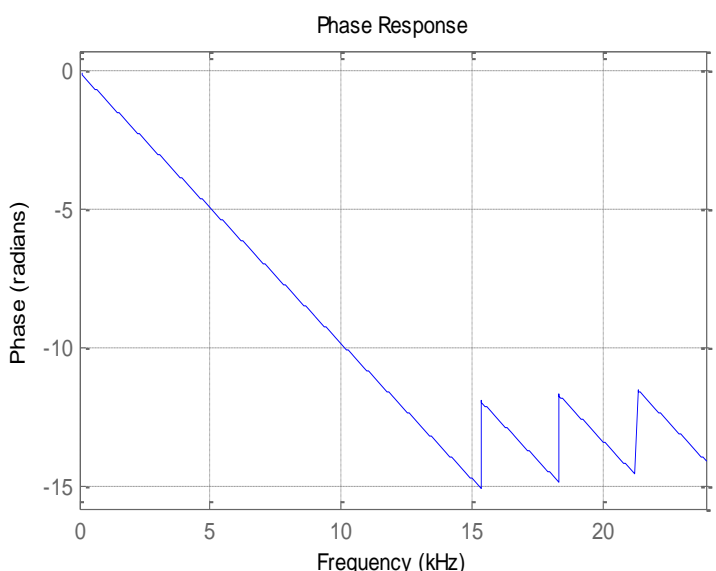

Fig.(15) Phase Response of Bartlett window

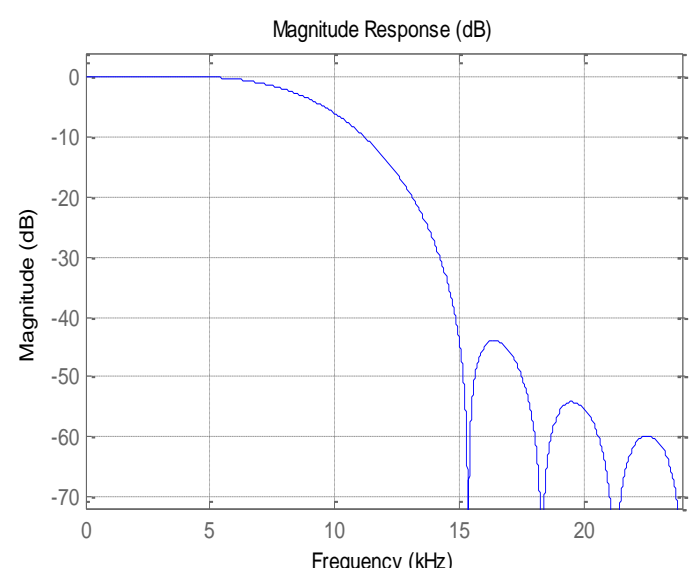

Fig.(16) Magnitude Response of Bartlett WIndow 


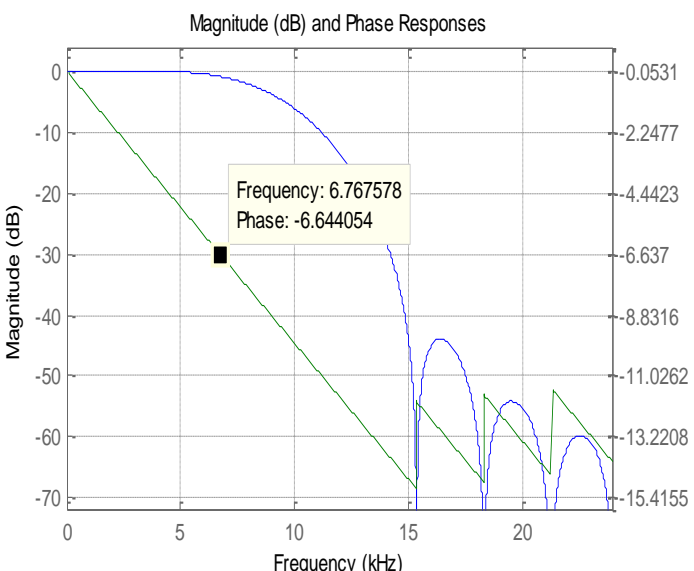

Fig.(17) Magnitude and Phase Response of Bartlett

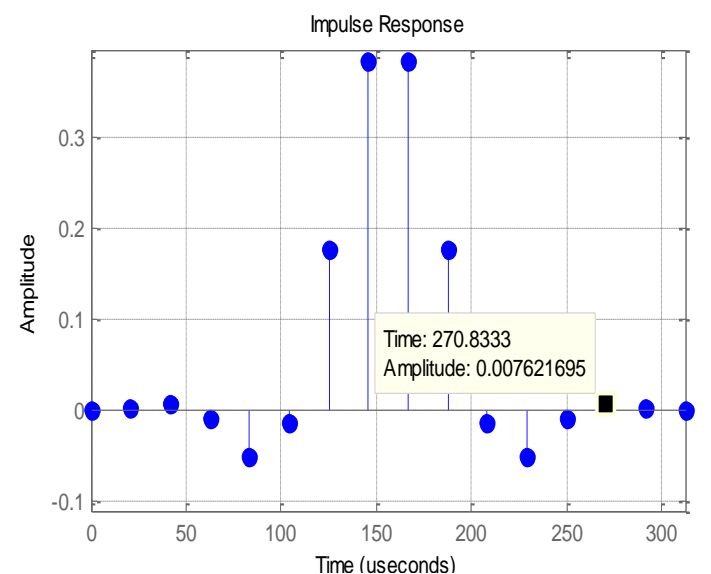

Fig.(18) Impulse Response of Bartlett window

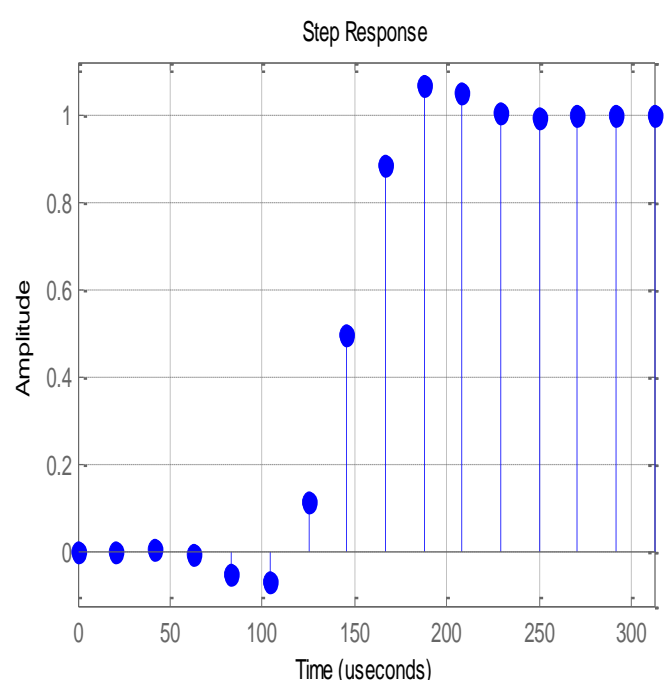

Fig.(19) Step Response of Bartlett window

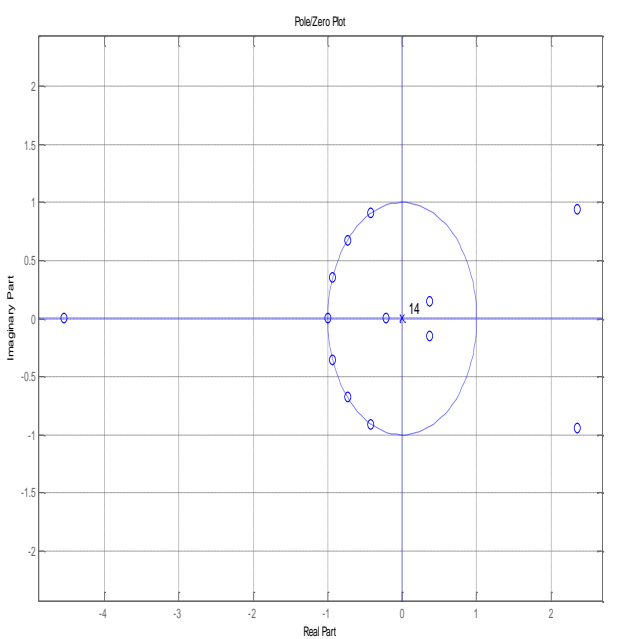

Fig.(20) Pole/Zero of Bartlett window

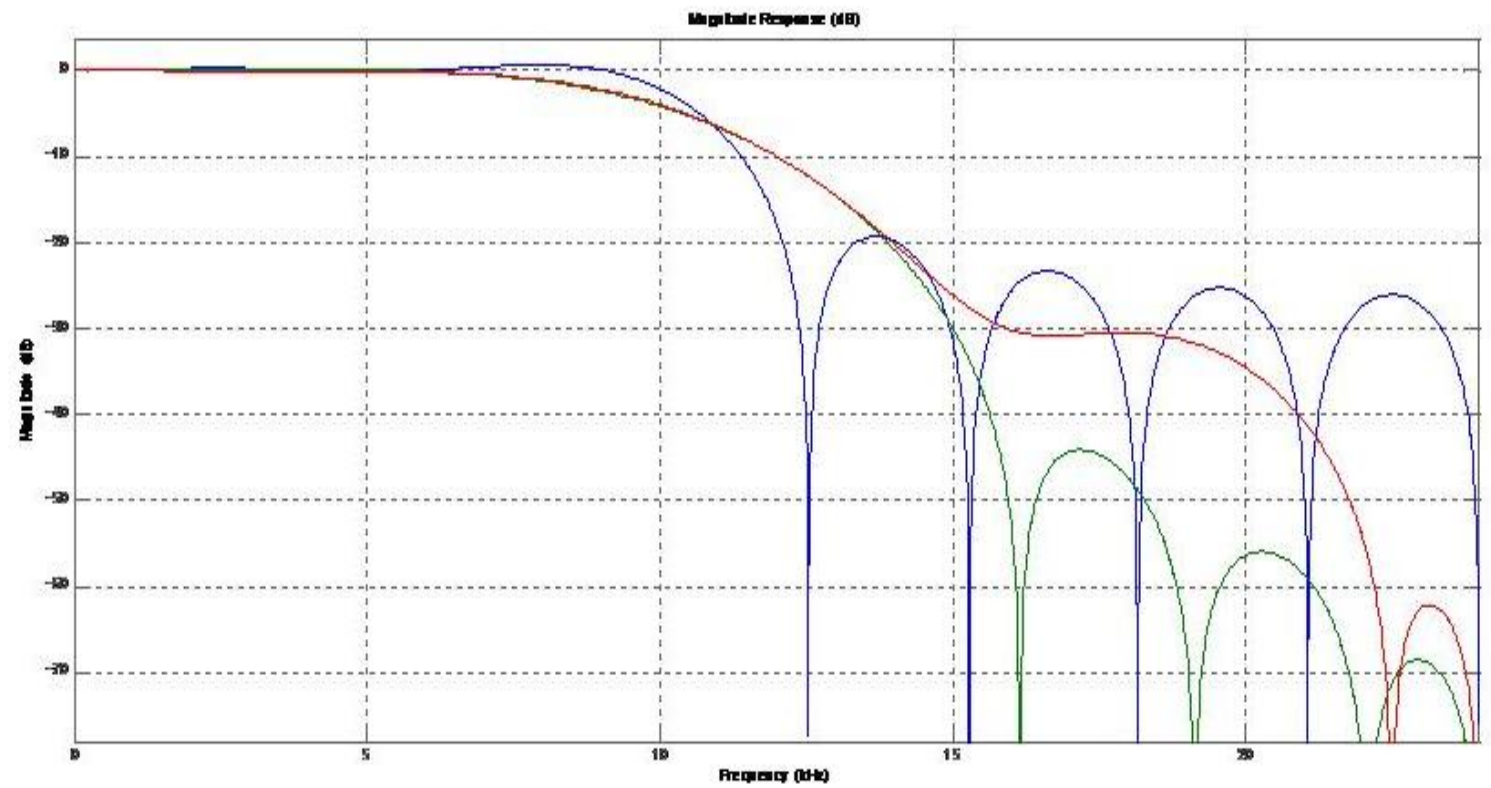

Fig.(21) Comparison of Magnitude Response of Rectangular Hanning and Bartlett window Tecnique 


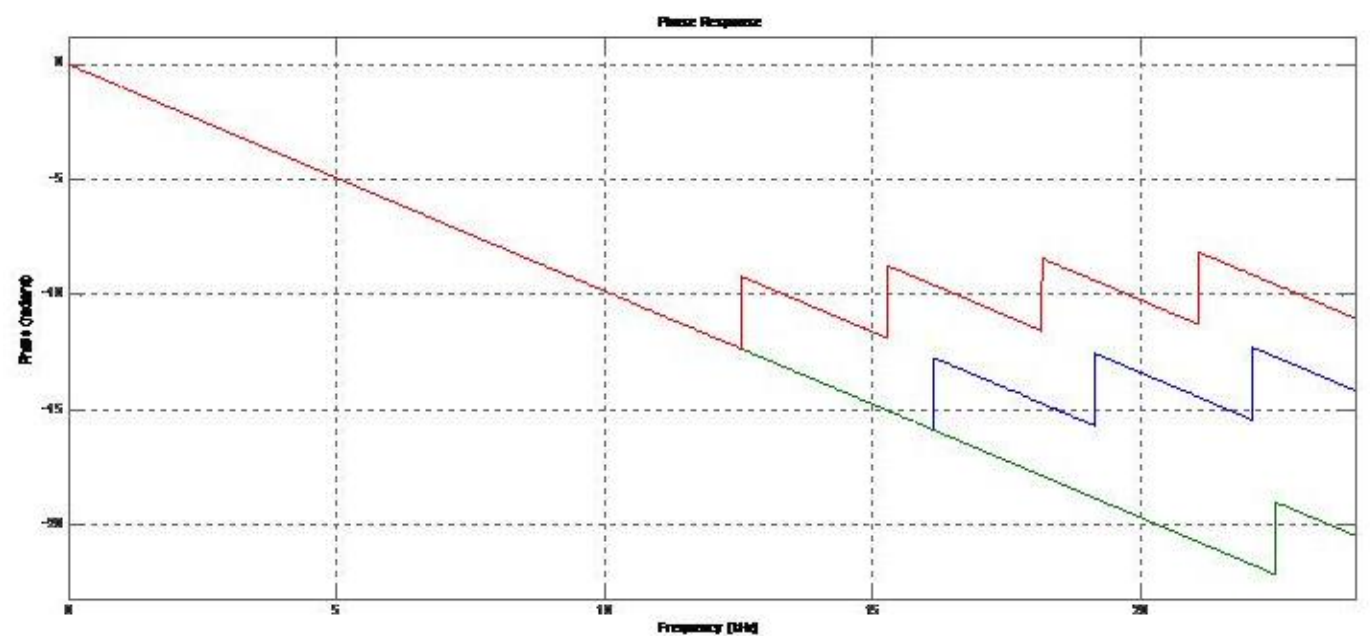

Fig.(22) Comparison of Phase Response of Rectangular Hanning and Bartlett Method

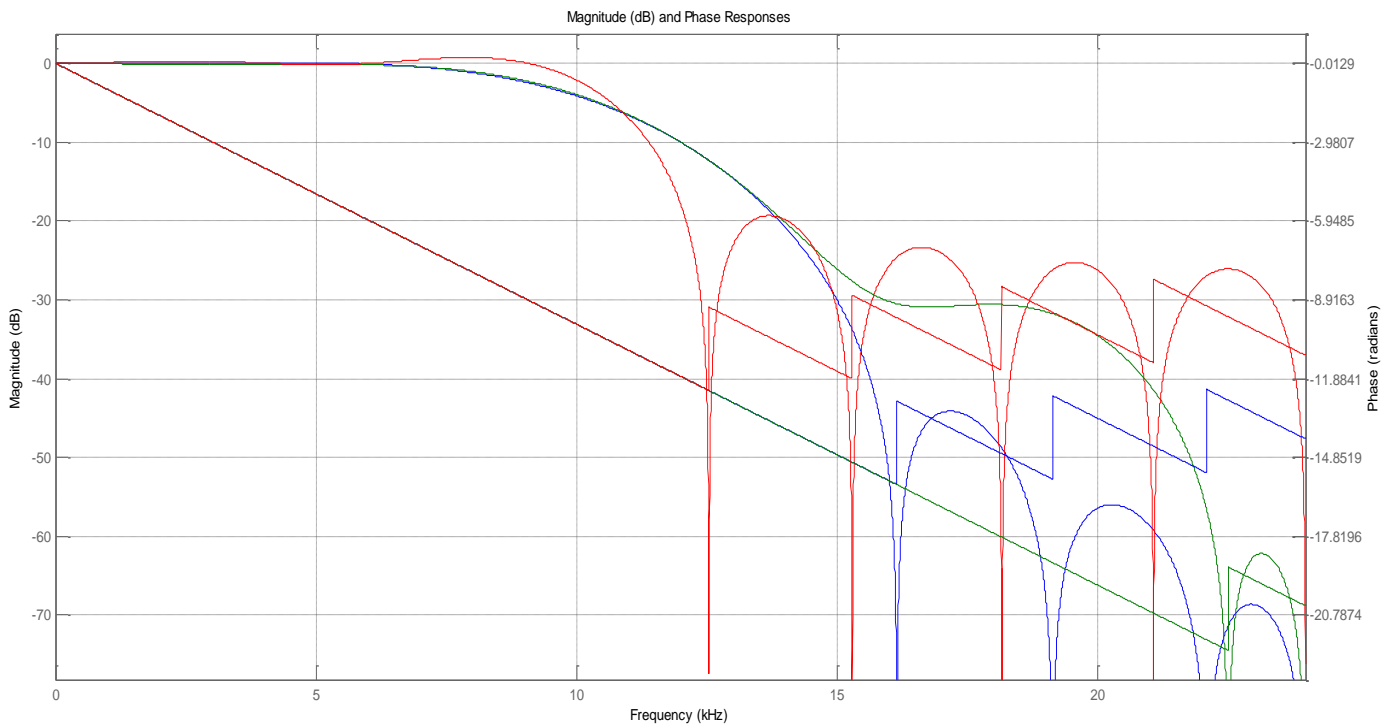

Fig.(24) Comparison of Magnitude and Phase Response of Rectangular Hanning and Bartlett Method

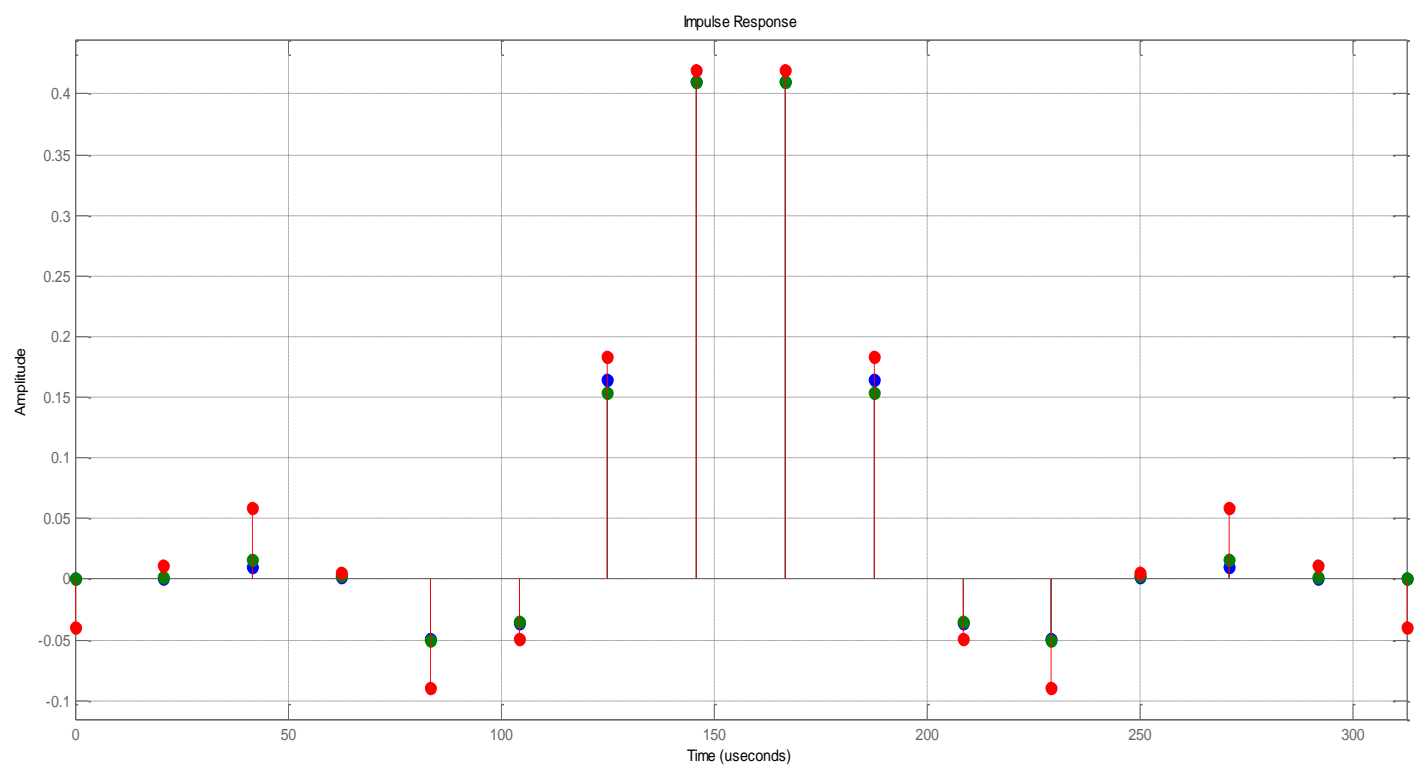

Fig(23) Comaprison of Impulse Response of Rectangular Hanning and Bartlett Method 


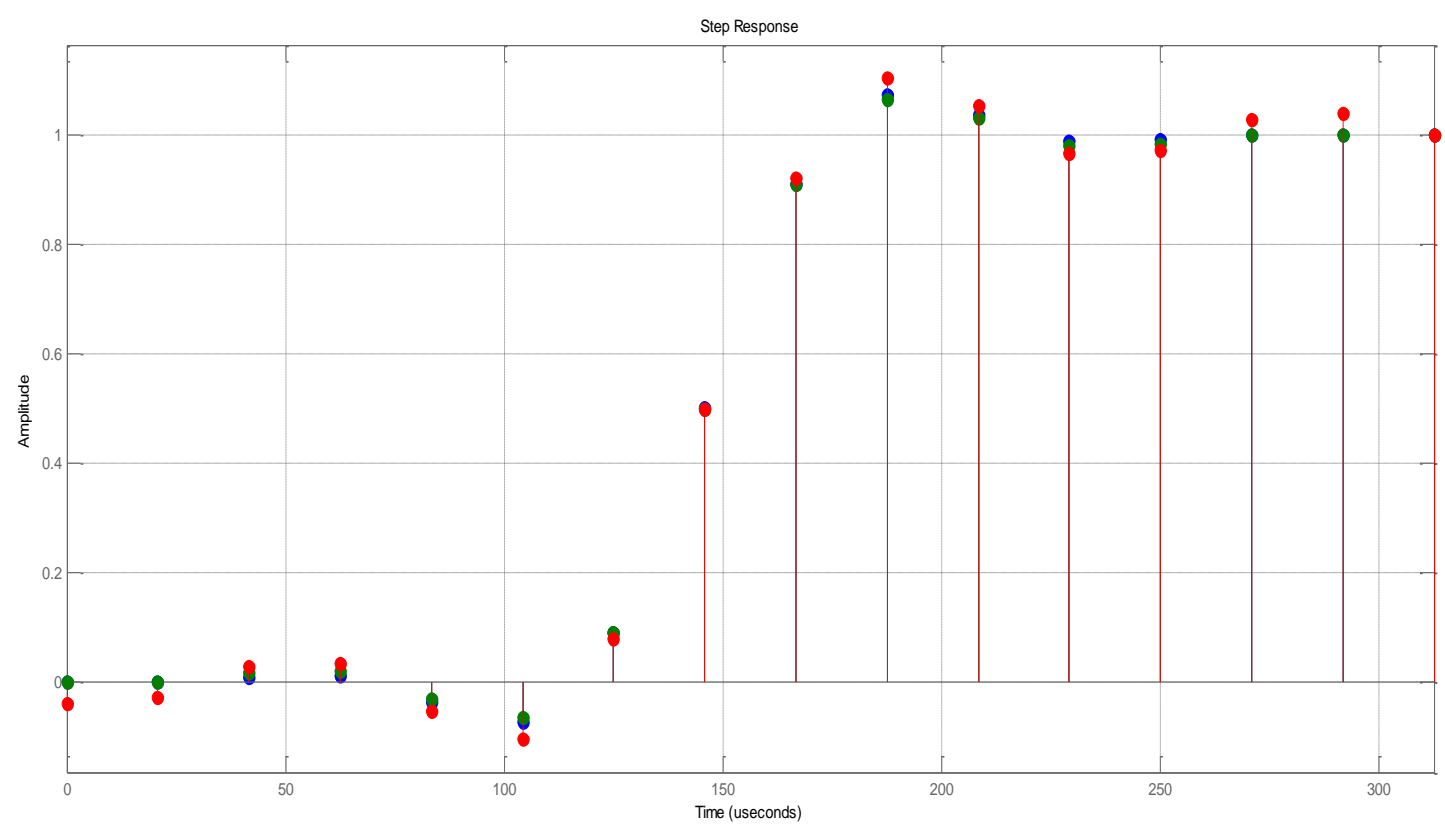

Fig.(24) Comparison of Step Response of Rectangular Hanning and Bartlett Method

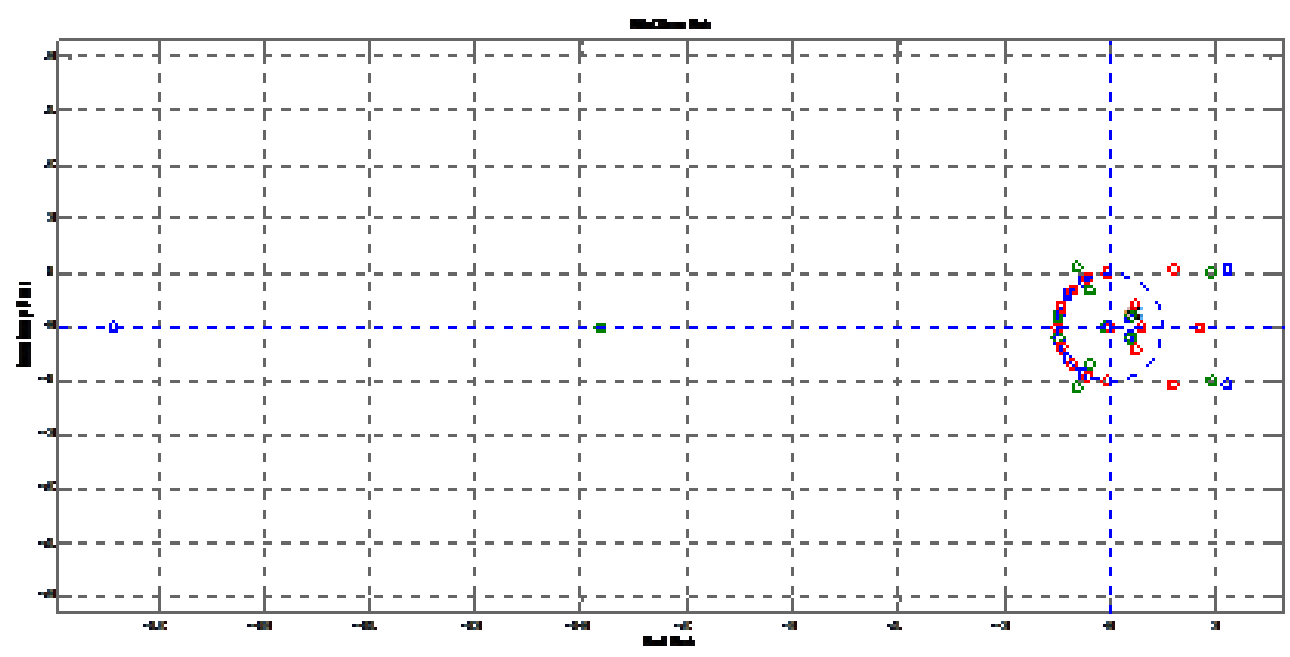

Fig.(25) Comparison of Pole/Zero Plot of Rectangular Hanning and Bartlett

\section{RESULT}

Simulation Result of MATLab

\begin{tabular}{|l|l|l|l|}
\hline Window Technique & Relative side lobe attenuation & Main lobe width (-3dB) Frequency & Leakage Factor \\
\hline RECTANGULAR & -31.5 & 0.20313 & $0.05 \%$ \\
\hline HANNING & -31.5 & 0.20313 & $0.05 \%$ \\
\hline Bartlett & -25.3 & 0.17939 & $0.42 \%$ \\
\hline
\end{tabular}

\section{CONCLUSIONS}

In this paper FIR low pass filter has been designed and simulated using Rectangular, Hanning and Kaiser Window technique has been compared, In Signal processing applications digital filters are more preferable than analog filters. The digital filters are easily designed and also easy to use in various types of signal filtering applications. The choice of technique to design the filter depends on the decision of designer whether $r$ to compromise accuracy of approximation or ease of design. 


\section{REFERENCES}

[1] 1Sriti Pandey, 2Kamlesh Sahu, 3Pooja Singh Chandel, 4 Pranay Kumar Rahi; 1, 2, 3 B.E.Scholar, 4Assistant Professor, 1,2,3,4 Department of Electrical \&Electronics Engineering, Institute of Technology Korba, Chhattisgarh, India (LOW PASS FILTER DESIGN AND ANALYSIS USING RECTANGULAR AND BARTLETT WINDOWS).

[2] Kurrey1, Varsha Nirala2, Pranay Kumar Rahi B.E. Scholar, 3Asst ProfessorDepartment of Electrical \& Electronics Engineering, Institute of Technology Korba, Chhattisgarh, India.

[3] J.G. Proakis and D. G. Manolakis, Digital Signal Processing Principles, Algorithms and Applications 3rd Edition Prentice- Hall, 2002.

[4] Sandeep Kaur, Sangeet Pal Kaur, "Design of FIR filter using hanning window, hamming window and modified hamming window", Vol 4 Issue 5, May 2015.

[5] S Salivahanan, C Gnanapriya, A Vallavaraj, "digital signal processing” Tata McGraw-Hill, second edition pp.3,443-448

[6] 1Pramod Yadav, 2Shankarlal Sahu, 3Vijendra Prajapati, 4Pranay Kumar Rahi 1,2,3BE Scholar, 4Assistant Professor 1,2,3,4Department of Electrical and Electronics Engineering 1,2,3,4Institute of Technology, Korba Chhattisgarh India( DESIGNING A LOW PASS FIR FILTER BY USING RECTANGULAR AND TAYLOR WINDOW)

[7] Carnegie Mellon, "Notes on FIR Filter Design Using Window Function”, Siganls and System (18-396).

\section{BIOGRAPHIES}

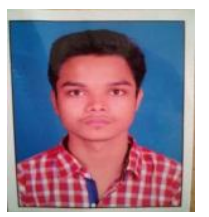

Vijendra Kumar Prajapati persuing bachelor of engineering in electrical and electronics engineering in 6th semester from institute of technology korba, Chhattisgarh swami Vivekananda university, chhattisgrh,india

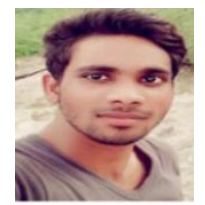

Dhanmohan Ratre persuing bachelor of engineering in electrical and electronics engineering in 6th semester from institute of technology korba, Chhattisgarh swami Vivekananda university, chhattisgrh,india

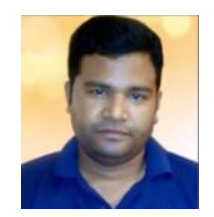

Pranay Kumar Rahi received the bachelor of technology degree in Electronics and Telecommunication engineering collage, guru ghashidas university, bilaspur Chhattisgarh, india in 2004, and persuing master of engineering in electronics and communication engineering from national institute of technical teacher's training \& research, Punjab university, Chandigarh, india. Presently working as assistant professor in department of electrical and electronics engineering, institute of technology, korba Ch.hattisgarh since 2008. He has authored more than 40 research publications and published journal paper and research papers in the leading international and national journal. This primary research interest includes digital signal processing, VLSI design, control systems and digital electronics and logic design. 